\title{
Transplantation of neural stem cells encapsulated in hydrogels improve functional recovery in a cauda equina lesion model
}

\section{Zhiyi Fu}

Shanghai Ninth People's Hospital

Huidong Wang

Shanghai Ninth People's Hospital

YujieWu ( $\nabla$ alphaf@163.com )

Shanghai Ninth People's Hospital,Shanghai Jiao Tong University School of Medicine

Tong Zhu

Shanghai Ninth People's Hospital

Research article

Keywords: cauda equina syndrome, cauda equina lesion model, neural stem cells, hydrogels, functional recovery

Posted Date: January 2nd, 2020

DOI: https://doi.org/10.21203/rs.2.19916/v1

License: (c) (i) This work is licensed under a Creative Commons Attribution 4.0 International License. Read Full License 


\section{Abstract}

Background This study explored the therapeutic effects of transplantation of neural stem cells (NSCs) encapsulated in hydrogels in a cauda equina lesion model.

Methods NSCs were isolated from neonatal dorsal root ganglion (DRG) and cultured in three-dimensional porous hydrogel scaffolds. Immunohistochemistry, transmission electron microscopy, Luxol fast blue staining, TUNEL assay were performed to detect the differentiation capability, ultrastructural and pathological changes, and apoptosis of NSCs. Furthermore, the functional recovery of sensorimotor reflexes was determined using the tail-flick test.

Results NSCs derived from DRG were able to proliferate to form neurospheres and mainly differentiate into oligodendrocytes in the three-dimensional hydrogel culture system. After transplantation of NSCs encapsulated in hydrogels, NSCs differentiated into oligodendrocytes, neurons or astrocytes in vivo . Moreover, NSCs engrafted on the hydrogels decreased apoptosis and alleviated the ultrastructural and pathological changes of injured cauda equina. Behavioral analysis showed that transplanted hydrogelencapsulated NSCs decreased the tail-flick latency and showed a neuroprotective role on injured cauda equina.

Conclusions Our results indicate transplantation of hydrogel-encapsulated NSCs promotes stem cell differentiation into oligodendrocytes, neurons or astrocytes and contributes to the functional recovery of injured cauda equina, suggesting that NSCs encapsulated in hydrogels may be applied for the treatment of cauda equina injury.

\section{Background}

Cauda equina syndrome (CES) is a neurological disease which is usually caused by central lumbar disc herniation. The consequences of CES, such as neuropathic pain, lower extremity dysfunction, and sexual dysfunction are extremely frustrating [1]. It greatly affects the patient's mental and physical condition and causes enormous economic impact to society. Although this disease has a low incidence in the population, ranging from 1:33,000 to 1:100,000 inhabitants, its sequelae still generate high public healthcare costs [2]. Current treatment strategies include application of corticosteroid, surgical stabilization and decompression, although effective but with unsatisfied therapeutic efficacy [3, 4]. Because of the poor self-repair ability of nerve tissue, especially those in the central nervous system, the therapeutic effects of surgery and neurotrophic drugs on cauda equina injury-induced CES is limited.

Neural stem cells (NSCs) can make copies of themselves and generate different mature cell types. They are promising candidate cells for neural transplantation treatment of neurological disorders, such as brain trauma, spinal cord injury, peripheral nerve injury [5-7]. Many studies indicate that stem cells foster host axons to grow into the grafted spinal cord [8-10]. In addition, induced pluripotent stem cells differentiate into astrocytes, neurons and oligodendrocytes and further improve functional recovery after spinal cord injury [11]. Nevertheless, application of stem cell transplantation therapy is limited by the poor 
cell survival at the injury site. Thus, development of novel approaches to maintain neural stem cell viability is important to achieve ideal therapeutic outcomes.

Recently, tissue engineering has been developed that could provide solutions to the problem of stem cell deaths during transplantation [12]. Biopolymer hydrogels are designed to promote stem cell survival after cerebral transplantation, exhibiting promising therapeutic role in central nervous system damage [13]. As previously reported by Singh et al, neural stem cells derived from adult dorsal root ganglia not only retain the multi-differentiation potential, but also have a tendency to differentiate into sensory neurons after transplantation, supporting that dorsal root ganglion neural stem cells (DRG NSCs) may be useful for repair of damaged cauda equina [14]. In the present study, NSCs were isolated from neonatal dorsal root ganglion (DRG), encapsulated in three-dimensional porous hydrogel scaffolds, and used to repair damaged cauda equina in a rat model of CES.

\section{Materials And Methods}

\section{Animals}

Male Sprague-Dawley rats weighing 200-250 g and aged 6-8 weeks were purchased from the Animal Center of the Second Military Medical University. The surgical interventions for animal experiments were approved by by the Ethical Committee of the Shanghai Jiao Tong University School of Medicine, and the animals were cared for in accordance with the Guide for the Care and Use of Laboratory Animals after the surgery.

\section{Culture of DRG-NSCs}

DRG were dissected from postnatal day 2 rats, mechanically dissociated in Hank's balanced saline solution, pH 7.4, and seeded in Dulbecco's modified Eagle's medium (DMEM)/F12 (Invitrogen) supplemented with $2 \%$ B27, $10 \mathrm{ng} / \mathrm{mL}$ epidermal growth factor (EGF), and $10 \mathrm{ng} / \mathrm{mL}$ bFGF. The cells were incubated in a humidified atmosphere with $5 \% \mathrm{CO}_{2}$ at $37^{\circ} \mathrm{C}$. The medium was changed every 3 days. The dissociated DRG cells formed clusters or neurospheres within 1-2 weeks. Afterwards, the neurospheres were collected, mechanically aspirated, and resuspended in the proliferation culture medium every 4 days to reduce the cell heterogeneity by maintaining small spheres (50-100 cells/sphere). After 3 generations of subcloning, the NSCs derived from the neurospheres were subjected to lentivirus transduction [15].

\section{Hydrogel preparation}

Hydrogels were purchased from Beaver Nano-Technologies Co., Ltd, China and were cross-linked with polyethylene glycol diacrylate. Solutions of this hydrogel form a transparent gel when mixed with a cross- 
linker over a period of 30 minutes. Then, NSCs were mixed with hydrogels to form a stem-cell-hydrogel complex.

\section{Model establishment and NSC transplantation}

Forty-eight SD rats were randomly divided into sham, CES model and NSCs transplantation groups. After anesthesia with chloral hydrate, laminectomy was performed at the lumbar 4 and a silicone band (10 $\mathrm{mm}$ long, $1 \mathrm{~mm}$ wide, and $1 \mathrm{~mm}$ thick) was placed under the laminae of the L5-6 vertebra to produce the CES animal model $[15,16]$. Sixteen rats were subjected to NSC transplantation, and another 16 rats were used as control. A sham operation was performed with a simple laminectomy but without contusion injury $(n=16)$.

For transplantation of NSCs, we removed the silicone 7 days after the compression injury and then intrathecally injected $12 \mu \mathrm{L} 0.5 \%$ hydrogels containing approximately 1000,000 NSCs (transduced with lentivirus) using a micropulled pipette connected to a Hamilton syringe. As control, $12 \mu \mathrm{L} 0.5 \%$ hydrogels containing no NSCs was injected into the subarachnoid space.

\section{Immunohistochemistry assay}

The cauda equina was extracted, placed in EDTA solution, and heated in an oven for antigen retrieval. Then, $15-\mu \mathrm{m}$ thick sections of the cauda equina around the lesion site were prepared longitudinally. The tissue sections were permeabilized with $0.2 \%$ Triton X-100 and blocked in blocking solution for $1 \mathrm{~h}$ at room temperature. After rinsing with PBS, the sections were incubated with primary antibodies against 04, $\beta$ III-tubulin, glial fibrillary acidic protein (GFAP) overnight at $4^{\circ} \mathrm{C}$ followed by incubation with secondary antibodies for $1 \mathrm{~h}$ at $37^{\circ} \mathrm{C}$. The slices were stained with Hoechst for 10 min and images were photographed by inverted fluorescence microscopy.

\section{Ultrastructural imaging}

For transmission electron microscopic (TEM) studies, the sections were fractured with liquid nitrogen and quenched in hydrogen peroxide solution. After rinsing in PBS, the sections were prepared for ultra-thin sectioning. Tissue sections were fixed in osmium tetroxide, dehydrated in ethanol, and embedded in resin. All samples were observed under transmission electron microscope (TEM).

\section{Luxol fast blue staining}


Tissue sections were stained with Luxol fast blue to detect myelin damage as previously described [17]. Briefly, the cauda equina sections were immersed in Luxol fast blue solution in an oven overnight. After rinsing in $95 \%$ ethanol and distilled water, the pathological changes of nerve fibers were observed under a light microscope.

\section{TdT-mediated dUTP-biotin nick end labeling (TUNEL) staining}

Apoptosis of cauda equina were measured by using a TUNEL detection kit according to the manufacturer's instructions (Sigma, USA). In brief, paraffin-embedded tissue sections (4-mm-thick) were dewaxed, rehydrated, and incubated with reaction mixture of terminal deoxynucleotidyl transferase for 1 h. After rinsing in PBS, the sections were incubated with biotinylated antibody and ABC complex, and photographed in a light microscope (Zeiss) equipped with a digital camera.

\section{Behavioral analysis}

For the tail-flick test, the rats were immobilized 20 min before the test, and the tail was placed over a slit. A beam of light from a projection lamp (voltage of $18.5 \mathrm{~V}$ ) was focused on the tail skin at the junction between the middle and distal $1 / 3$ of the tail. The latency to respond was recorded with a maximal $15 \mathrm{~s}$ radiant heat stimulus [15].

\section{Statistical analysis}

Experimental data are presented as mean \pm SD. One-Way ANOVA was used for comparison of different groups. Results were considered statistically significant when the $P$ value was less than 0.05 .

\section{Results}

\section{Characterization of NSCs in hydrogel scaffolds}

NSCs were isolated from the neonatal rat DRG and cultured in proliferation culture medium for different days. We observed that many cells were floated in the medium and formed neurospheres (Figure 1A). Then, NSCs were successfully grown in $0.25 \%$ and $0.5 \%$ hydrogels (Figure $1 \mathrm{~A}$ ). Immunohistochemical staining showed that cells were positive for nestin and P75R, two markers of NSCs (Figure 1B).

\section{Differentiation of NSCs in hydrogel scaffolds}


To more easily track cells, NSCs were transfected with lentivirus vectors carrying green fluorescent protein (GFP) and cultured in differentiation medium (Figure 2A). After 7 days of differentiation in vitro, the neural progenitors in the spheres attached to the hydrogel scaffolds and differentiated into different types of cells. Immunohistochemical staining showed that most of the GFP labelled NSCs differentiated into 04+ oligodendrocytes, and only a very few cells differentiated into S100+ Schwann cells, $\beta$ III-tubulin+ neurons and GFAP+ astrocytes (Figure 2B).

\section{Differentiation of NSCs following transplantation in the injured cauda equina}

Next, we successful established the rat model of cauda equina injury and transplanted hydrogelencapsulated NSCs (Figure 3A). To detect the viability of the transplanted NSCs in the cauda equina, the frozen sections were imaged by confocal microscopy after 7 days of transplantation. As expected, GFPpositive grafted NSCs were present in the injured cauda equina (Figure $3 \mathrm{~B}$ ). Furthermore, immunostaining of the sagittal sections of the cauda equina showed that GFP-positive cells were co-stained with 04 , $\beta$ IIItubulin or GFAP, but not with S100, suggesting that NSCs differentiated into oligodendrocytes, neurons or astrocytes in vivo (Figure 4).

\section{Functional recovery following NSC transplantation encapsulated in three-dimensional hydrogels}

To explore the regenerative effect of the transplanted NSCs, the functional recovery of sensorimotor reflexes was determined using the tail-flick test. Fourteen days after transplantation, rat bilateral L5-6 DRG tissues were isolated and subject to TUNEL staining. Results showed that cauda equina injury led to a significant increase in apoptotic cells compared to sham group $(P<0.01)$. By contrast, NSCs transplantation with three-dimensional hydrogels obviously decreased the apoptosis rate $(P<0.05)$ (Figure 5A). Two weeks after surgery, LFB staining and TEM analysis showed cauda equina nerve fibers in an organized state, normal axons, and intact myelin sheath. However, compression of cauda equina resulted in disorganized nerve fibers, swollen axons and myelin sheaths, and demyelination. These observations were alleviated after transplantation of NSCs encapsulated in hydrogels (Figure 5A). Additionally, G-ratio (inner diameter/outer diameter of myelinated axons) was significantly higher in model group than sham group, and then decreased in the grafted NSCs animals (Figure 5B).

The rat model procedure was described and modified by our research team, which has been successfully used in many studies. When we established the model, we proved that: at day 1,3 , and 7 post compression of cauda equina, we observed higher the tail flick latency (TFL) of the compression group than that of the sham group. The compression group was significantly prolonged. After 7 days, immunofluorescence staining showed dense and organized cauda equina nerve fibers, no swollen axons, and intact myelin in the sham group. However, in the compression group, we observed disorganized cauda equina nerve fibers, swollen axons and demyelinated myelin. 
Three weeks after transplantation, animals with cauda equina injury exhibited a significant increase in tail-flick latency compared with those in the sham group. However, transplantation of hydrogelencapsulated NSCs partly decreased the tail-flick latency, exhibiting a neuroprotective activity on injured cauda equina (Figure 6).

\section{Discussion}

CES is a rare neurological disorder characterized low back pain, muscle weakness, and sensory disturbance [1]. At the cellular level, DRG cells appear disorganized with some apoptotic bodies. In addition, CES will cause demyelination and swelling of myelin. Because of the poor self-repair ability of nerve tissue, especially those in the central nervous system, the therapeutic effects of surgery and neurotrophic drugs on cauda equina injury-induced CES is limited. Patients often have residual bladder and sexual dysfunction, and skin sensory disorder in the sella area. Although this disease has a low incidence in the population, ranging from $1: 33,000$ to $1: 100,000$ inhabitants, its sequelae still generate high public healthcare costs[2]. Thus, development of novel approaches to maintain neural stem cell viability is important to achieve ideal therapeutic outcomes. The functional recovery of CES is not satisfied because of the failure of axon regeneration and the nerve damage.

Because the cauda equina is different from the spinal cord, it is impossible to transplant the neural stem cells into the solid tissues. Our previous study showed that GFP-NSCs survived in the cerebrospinal fluid around the damaged cauda equina after intrathecal transplantation, but the surviving time was very short, i.e., only one week [15]. Based on the previous study, hydrogels were used to localize neural stem cells to the injured cauda equina to promote the axon regeneration and remyelination of damaged cauda equina, eventually achieving the goal of neuron preservation and functional repair.

In the present study, we applied tissue engineering technology combined with NSCs transplantation, and explored the potential therapeutic effects on cauda equina injury. Consequently, our study demonstrated that transplantation of hydrogel-encapsulated NSCs significantly promoted the differentiation of stem cells and improved the functional recovery of injured cauda equina.

NSCs have shown promising and beneficial effects in the therapy of neurological disorders, such as spinal cord injury, brain trauma, and cauda equina lesion [18]. Accumulating evidence demonstrates that transplanted NSCs successfully survive in the injured tissues and integrate into the host brain to achieve the functional recovery [19]. Moreover, the pluripotency of DRG has been reported by several research groups, including our group $[15,20,21]$. The sensory branch in the cauda equina is composed of the central processes of DRG neurons. Thus, DRG-NSCs were used to repair the damaged cauda equina because the homology of DRG-NSCs with cauda equina. The mechanisms by which NSCs exert their neuroprotective effects have begun to be elucidated. Increasing studies have shown that NSCs may synthesize a variety of neurotrophic cytokines stimulating nerve growth, such as vascular endothelial growth factor (VEGF), brain derived neurotrophic factor (BDNF), and nerve growth factor (NGF) [22, 23]. Previous studies showed that deficiency of endogenous neurotrophins is associated with poor neuronal 
survival and cell death [24]. BDNF has very extensive neurotrophy and can maintain the survival of various kinds of neurons and directly promote their axon growth [25]. Following a cervical spinal cord injury, administration of BDNF into the site of spinal cord injury promoted axonal regeneration and prevented axotomy-induced atrophy and/or death of rubrospinal neurons [26, 27]. Furthermore, cell transplantation may also enhance endogenous repair processes including neurogenesis, axonal sprouting, and angiogenesis $[28,29]$. However, NSCs application is limited due to the poor cell survival in host tissues. In our study, NSCs were successfully isolated and cultured in hydrogels. Moreover, we found that NSCs could differentiate into oligodendrocytes, Schwann cells, neurons and astrocytes.

Tissue engineering may provide solutions to the challenges of stem cell death and damage associated with transplantation [30]. Biopolymer hydrogels can promote stem cell survival, enhance stem cell engraftment, and minimize wound scar formation [13]. Published studies have shown that hydrogels alter the survival and differentiation of stem cells both in vitro and in vivo [31, 32]. In the present study, we isolated NSCs from neonatal DRG to repair damaged cauda equina in a rat model of lumbar spinal canal stenosis. As a result, hydrogel-encapsulated NSCs presented high viability in the injured cauda equina and mainly differentiated to oligodendrocytes. Oligodendrocytes are known to be susceptible to spinal cord contusion and loss of oligodendrocytes may induce demyelination, disturb the functional recovery of damaged nerve tissues, and damage the conductive capacity of sensory nerves [33]. Therefore, stem cell transplantation is helpful to improve myelination and enhance functional recovery after CNS injury [34]. To evaluate the neuroprotective role of the hydrogel encapsulated NSCs, the tail-flick test was performed to measure the functional recovery of sensorimotor reflexes. As expected, NSCs engrafted on the hydrogels obviously decreased apoptosis of injured cauda equina tissue. Moreover, cauda equina nerve fibers presented an organized state, normal axons, and intact myelin sheath. Additionally, transplanted hydrogel-encapsulated NSCs decreased the tail-flick latency and showed a neuroprotective role on injured cauda equina.

In summary, our study demonstrates that transplantation of hydrogel-encapsulated NSCs enhances the viability of transplanted cells, promotes stem cell differentiation into oligodendrocytes, thereby contributing to the functional recovery of injured cauda equina. These results implied that NSCs encapsulated in three-dimensional hydrogels may be used for the treatment of cauda equina disorder.

\section{Conclusions}

Our results indicate transplantation of hydrogel-encapsulated NSCs promotes stem cell differentiation into oligodendrocytes, neurons or astrocytes and contributes to the functional recovery of injured cauda equina, suggesting that NSCs encapsulated in hydrogels may be applied for the treatment of cauda equina injury.

\section{Declarations}

\section{Availability of data and materials}


All data generated or analyzed during this study are included in this published article.

\section{Abbreviations}

CES]

Cauda equina syndrome

NSCs:

neural stem cells

DRG:

dorsal root ganglion

GFAP:

glial fibrillary acidic protein

\section{TEM:}

transmission electron microscopic

GFP:

green fluorescent protein

\section{Acknowledgements}

Not applicable.

\section{Funding}

This research is supported by the National Natural Science Foundation of China (grant \#81400997), Shanghai Municipal Commission of Health and Family Planning (grant \#201440326).

\section{Author information}




\section{Affiliations}

Shanghai Key Laboratory of Orthopaedic Implants, Department of Orthopaedic Surgery, Shanghai Ninth People's Hospital, Shanghai Jiao Tong University School of Medicine, 639 Zhizaoju Rd, Shanghai 200011, China.

\section{Author Contributions Statement}

Zhiyi Fu Manuscript Preparation, Literature Search, Funds Collection

Huidong Wang Data Collection

Yujie Wu Study Design, Data Interpretation

Tong Zhu Statistical Analysis

Corresponding author

Correspondence to Yujie Wu

\section{Ethics declarations}

\section{Ethics approval and consent to participate}

The study protocol was approved by by the Ethical Committee of the Shanghai Jiao Tong University School of Medicine. Informed consent was provided by all participating individuals.

\section{Consent for publication}

Not applicable.

\section{Competing interests Statement}

No competing interests.

\section{References}


1. Ma B, Wu H, Jia LS, et al. Cauda equina syndrome: a review of clinical progress. Chin Med J (Engl). 2009;122:1214-1222.

2. Dias ALN, Araújo FF, Cristante AF, et al. Epidemiology of cauda equina syndrome. What changed until 2015. Rev Bras Ortop. 2017;53(1):107-112.

3. Li X, Dou Q, Hu S, et al. Treatment of cauda equina syndrome caused by lumbar disc herniation with percutaneous endoscopic lumbar discectomy. Acta Neurol Belg. 2016;116(2):185-190.

4. Korse NS, Pijpers JA, Zwet EV, et al. Cauda Equina Syndrome: presentation, outcome, and predictors with focus on micturition, defecation, and sexual dysfunction. Eur Spine J. 2017;26(3):894-904.

5. Imitola J, Raddassi K, Park KI, et al. Directed migration of neural stem cells to sites of CNS injury by the stromal cell-derived factor 1 alpha/CXC chemokine receptor 4 pathway. Proc Natl Acad Sci U S A. 2004;101(52):18117-18122.

6. Abe K. Therapeutic potential of neurotrophic factors and neural stem cells against ischemic brain injury. J Cereb Blood Flow Metab. 2000;20(10):1393-1408.

7. Haus DL, López-Velázquez L, Gold EM, et al. Transplantation of human neural stem cells restores cognition in an immunodeficient rodent model of traumatic brain injury. Exp Neurol. 2016;281:1-16.

8. Marei HE, Shouman Z, Althani A, et al. Differentiation of human olfactory bulb-derived neural stem cells toward oligodendrocyte. J Cell Physiol. 2018;233(2):1321-1329.

9. Zhang LQ, Zhang WM, Deng L, et al. Transplantation of a Peripheral Nerve with Neural Stem Cells Plus Lithium Chloride Injection Promote the Recovery of Rat Spinal Cord Injury. Cell Transplant. 2018;27(3):471-484.

10. Méndezolivos EE, Muñoz R, Larraín J. Spinal Cord Cells from Pre-metamorphic Stages Differentiate into Neurons and Promote Axon Growth and Regeneration after Transplantation into the Injured Spinal Cord of Non-regenerativeXenopus laevisFroglets. Front Cell Neurosci. 2017;11:398.

11. Levison SW, Druckman SK, Young GM, et al. Neural stem cells in the subventricular zone are a source of astrocytes and oligodendrocytes, but not microglia. Dev Neurosci. 2003;25(2-4):184-196.

12. Wang A, Tang Z, Park IH, et al. Induced pluripotent stem cells for neural tissue engineering. Biomaterials. 2011;32(22):5023-5032.

13. Chen XY, Zhuang SJ, Hou XM, et al. Research Progress on Tissue Engineering of Neural Stem Cells. Adv Mat Res. 2012;535-537:1104-1107.

14. Singh RP, Cheng YH, Nelson P, et al. Retentive multipotency of adult dorsal root ganglia stem cells. Cell Transplant. 2009;18(1):55-68.

15. Fu ZY, Shi JG, Liu N, et al. Differentiation of neonatal dorsal root ganglion-derived neural stem cells into oligodendrocytes after intrathecal transplantation into a cauda equina lesion model. Genet Mol Res. 2013;12(4):6092-6102.

16. Liu X, Fu Z, Wu Y, et al. Neuroprotective effect of hydrogen sulfide on acute cauda equina injury in rats. Spine J. 2016;16(3):402-7. 
17. Kim JH, Budde MD, Liang HF, et al. Detecting axon damage in spinal cord from a mouse model of multiple sclerosis. Neurobiol Dis. 2006;21(3):626-632.

18. Pluchino $S$, Zanotti L, Deleidi M, et al. Neural stem cells and their use as therapeutic tool in neurological disorders. Brain Res Rev. 2005;48(2):211-219.

19. Uchida K, Momiyama T, Okano H, et al. Potential functional neural repair with grafted neural stem cells of early embryonic neuroepithelial origin. Neurosci Res. 2005;52(3):276.

20. Ogawa R, Fujita K, Ito K. Mouse embryonic dorsal root ganglia contain pluripotent stem cells that show features similar to ES cells and induced pluripotentstem Biol Open. 2017;6(5):602-618.

21. Hu H, Ding Y, Mu W, et al. DRG-Derived Neural Progenitors Differentiate into Functional Enteric Neurons Following Transplantation in the Postnatal Colon. Cell Transplant. 2019;28(2):157-169.

22. Salewski RP, Mitchell RA, Li L, et al. Transplantation of Induced Pluripotent Stem Cell-Derived Neural Stem Cells Mediate Functional Recovery Following Thoracic Spinal Cord Injury Through Remyelination of Axons. Stem Cells Transl Med. 2015;4(7):743-754.

23. Upadhyay G, Shankar S, Srivastava RK. Stem Cells in Neurological Disorders: Emerging Therapy with Stunning Hopes. Mol Neurobiol. 2015;52(1):610-625.

24. Nakamura M, Bregman BS. Differences in neurotrophic factor gene expression profiles between neonate and adult rat spinal cord after injury. Exp Neurol. 2001;169(2):407-15.

25. McCall J, Weidner N, Blesch A. Neurotrophic factors in combinatorial approaches for spinal cord regeneration. Cell Tissue Res. 2012;349(1):27-37.

26. Liu Y, Himes BT, Murray M, et al. Grafts of BDNF-producing fibroblasts rescue axotomized rubrospinal neurons and prevent their atrophy. Exp Neurol. 2002;178(2):150-64.

27. Tan J, Shi J, Shi G, et al. Changes in compressed neurons from dogs with acute and severe cauda equina constrictions following intrathecal injection of brain-derived neurotrophic factor-conjugated polymer nanoparticles. Neural Regen Res. 2013;8(3):233-43.

28. Andres RH, Horie N, Slikker W, et al. Human neural stem cells enhance structural plasticity and axonal transport in the ischaemic brain. Brain. 2011;134(Pt6):1777-1789.

29. Daadi MM, Davis AS, Arac A, et al. Human neural stem cell grafts modify microglial response and enhance axonal sprouting in neonatal hypoxic-ischemic brain injury. Stroke. 2010;41(3):516-523.

30. Kim H, Cooke MJ, Shoichet MS. Creating permissive microenvironments for stem cell transplantation into the central nervous system. Trends Biotechnol. 2012;30(1):55-63.

31. Führmann T, Tam RY, Ballarin B, et al. Injectable hydrogel promotes early survival of induced pluripotent stem cell-derived oligodendrocytes and attenuates longterm teratoma formation in a spinal cord injury model. Biomaterials. 2016;83:23-36.

32. Naghdi P, Tiraihi T, Ganji F, et al. Survival, proliferation and differentiation enhancement of neural stem cells cultured in three-dimensional polyethylene glycol-RGD hydrogel with tenascin. J Tissue Eng Regen Med. 2016;10(3):199-208. 
33. Nistor GI, Totoiu MO, Haque N, et al. Human embryonic stem cells differentiate into oligodendrocytes in high purity and myelinate after spinal cord transplantation. Glia. 2005;49(3):385-396.

34. Armstrong RC, Mierzwa AJ, Sullivan GM, et al. Myelin and Oligodendrocyte Lineage Cells in White Matter Pathology and Plasticity after Traumatic Brain Injury. Neuropharmacology. 2016;110(Pt8):654-659.

\section{Figures}

A
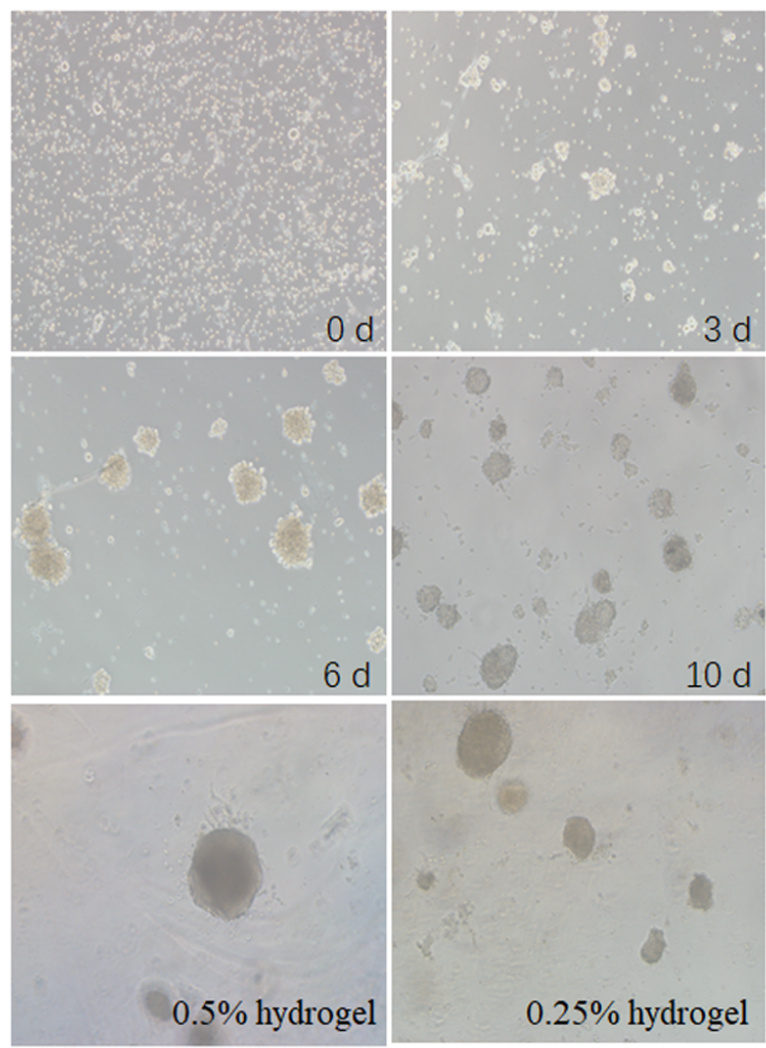

B

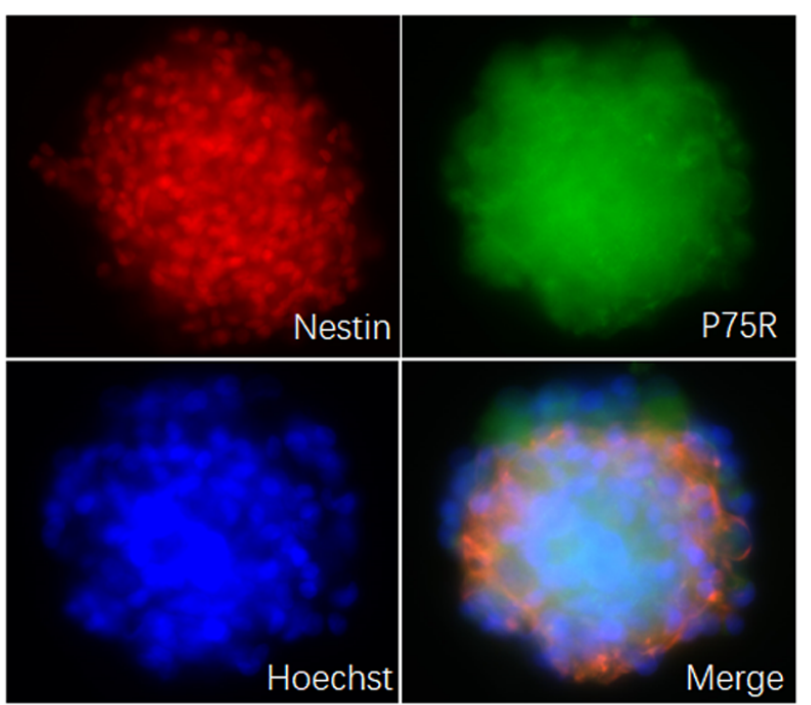

\section{Figure 1}

Characterization of NSCs in hydrogel scaffolds NSCs were isolated from the neonatal rat DRG and cultured in proliferation culture medium for different time points at $0,3,6$, and 10 days (A). For identification of NSCs, stem cells were immunohistochemically stained with antibodies against nestin and P75R, and nucleus was stained with Hoechst (B). 
A

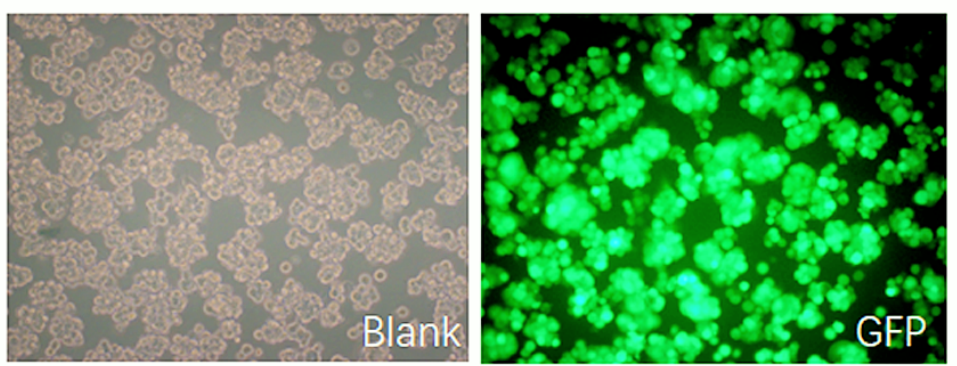

B

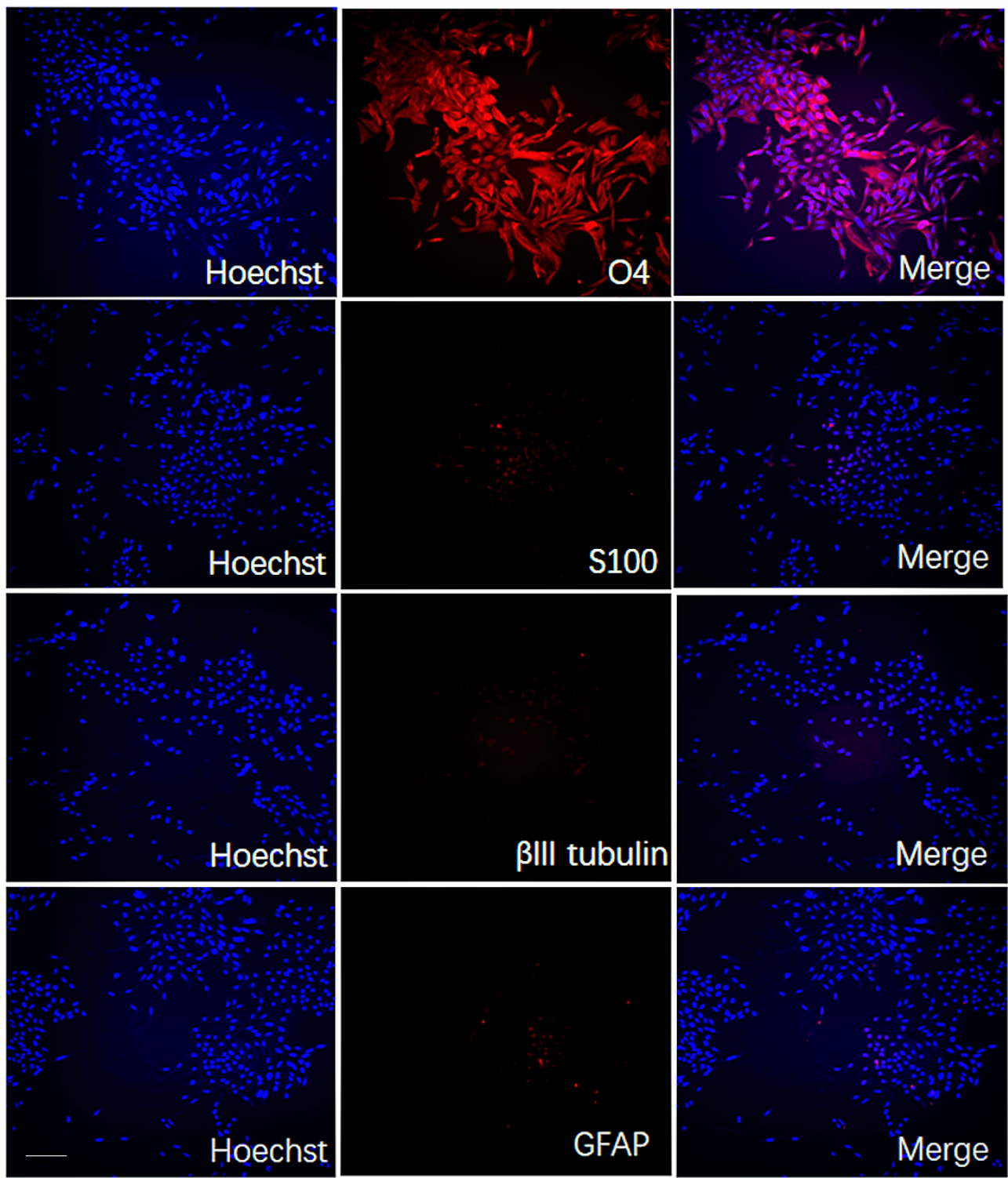

\section{Figure 2}

Differentiation of NSCs in vitro NSCs were transfected with lentivirus vectors carrying green fluorescent protein (GFP) and cultured in differentiation medium (A). After 7 days of differentiation, stem cells were immunohistochemically stained with antibodies against 04, S100, Blll-tubulin, and GFAP, and nucleus was stained with Hoechst (B). Scale bar=50 $\mathrm{mm}$. 
A
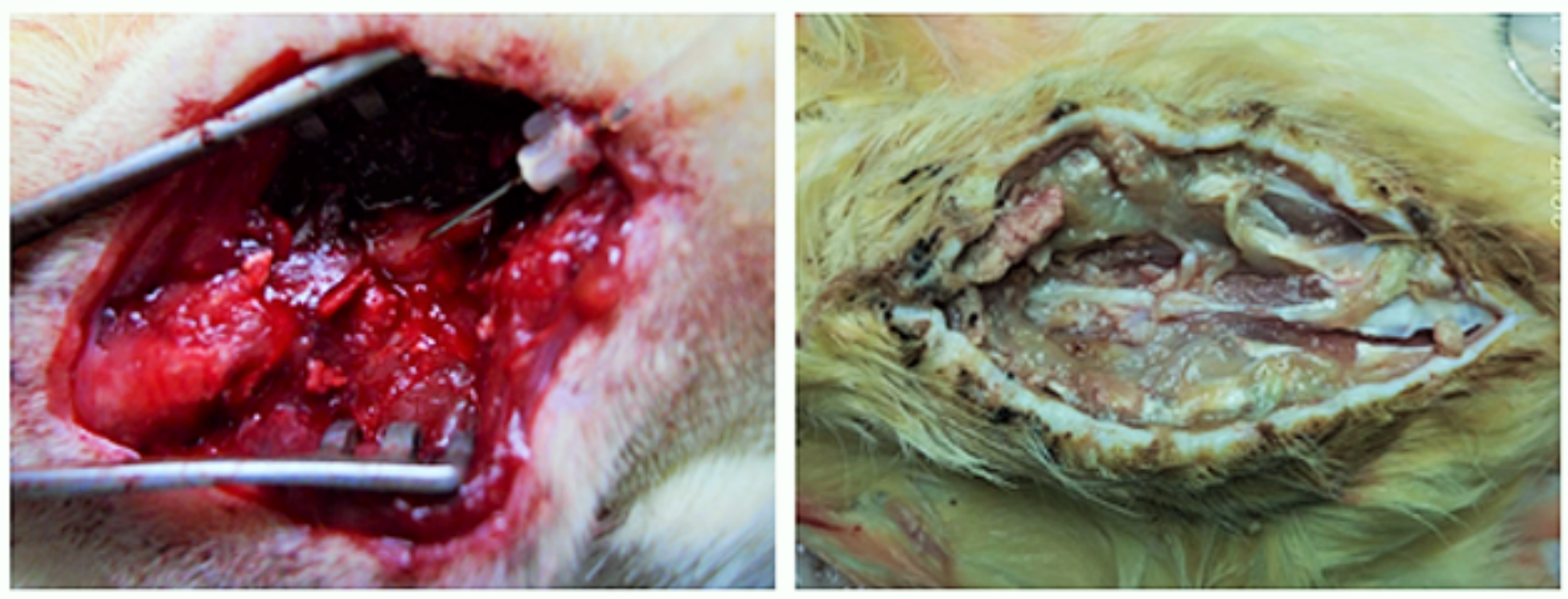

B

Hydrogel

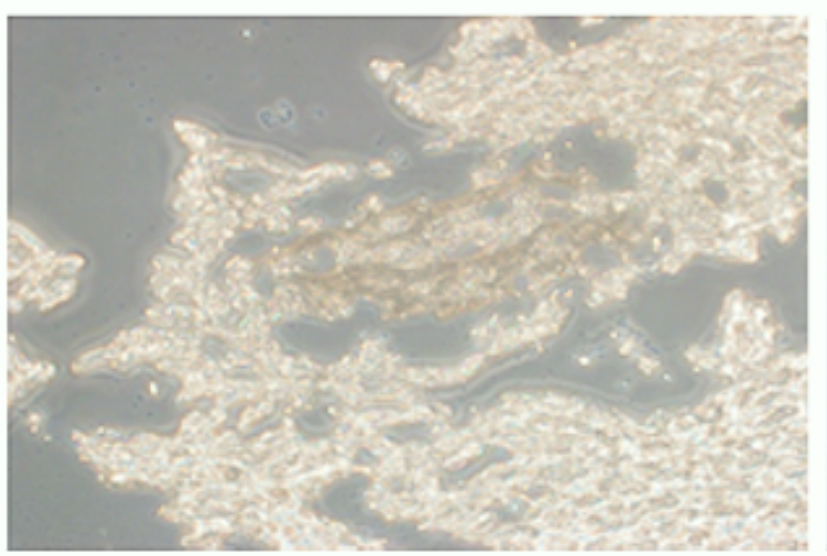

GFP

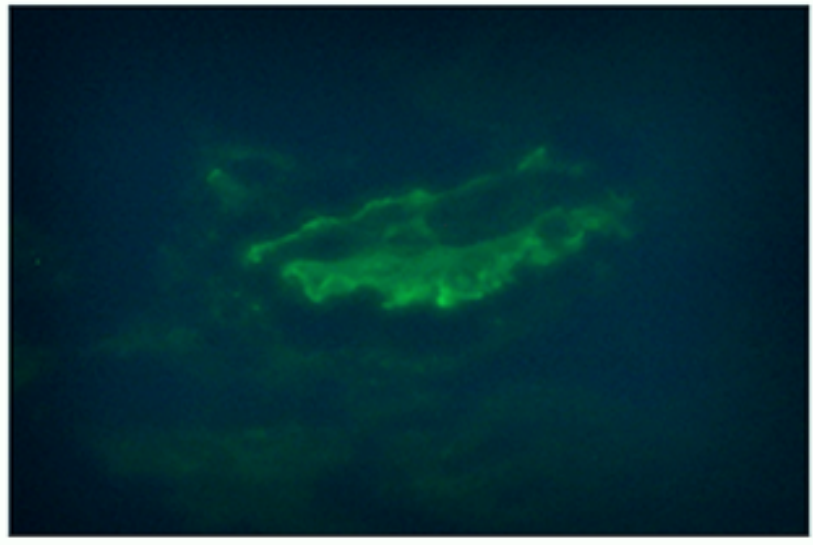

\section{Figure 3}

Transplantation of NSCs in the injured cauda equina A rat model of cauda equina injury were established and then transplanted with hydrogel-encapsulated NSCs (A). After 7 days of transplantation, the frozen sections were imaged and GFP-positive grafted NSCs were observed in the injured cauda equina (B). 

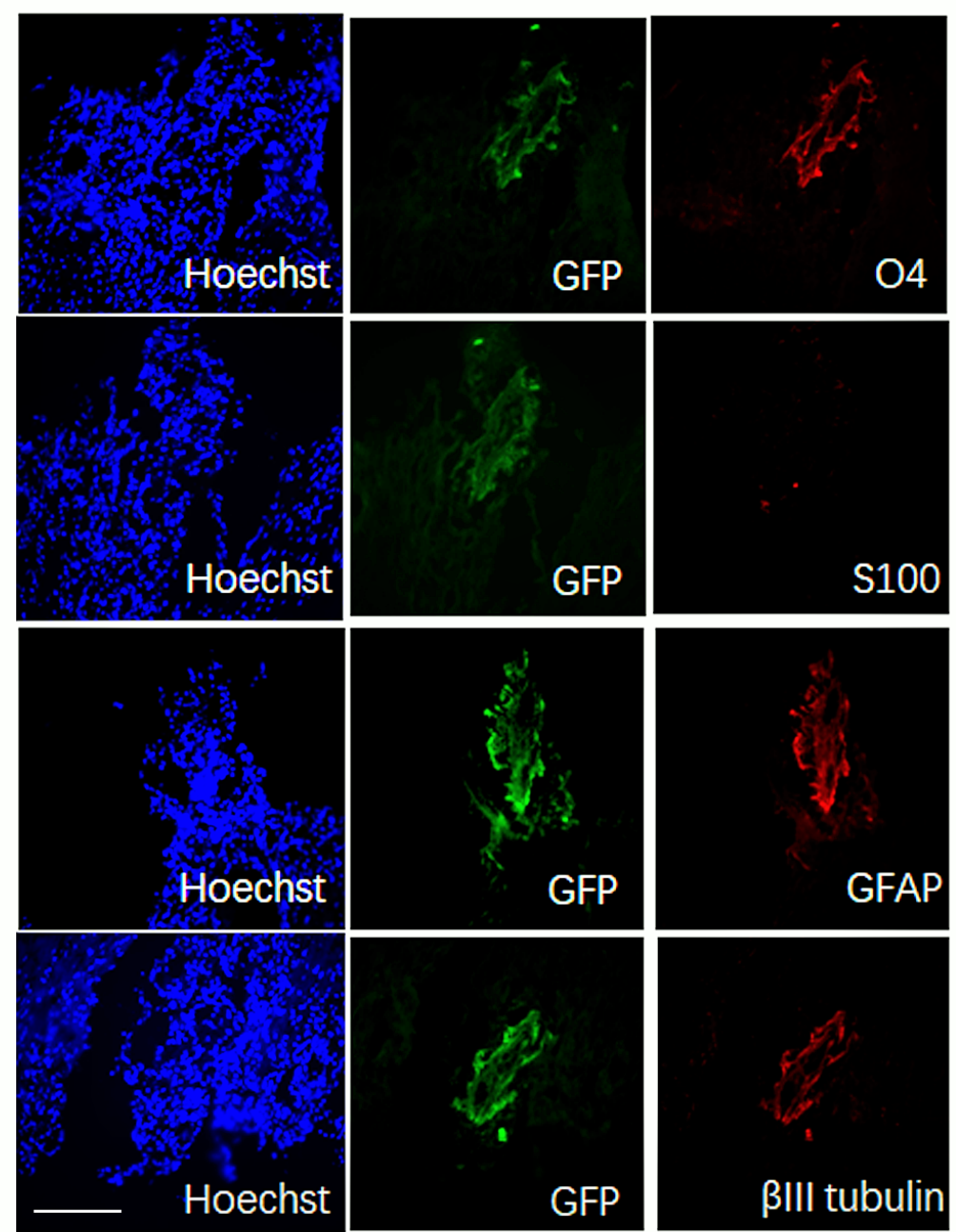
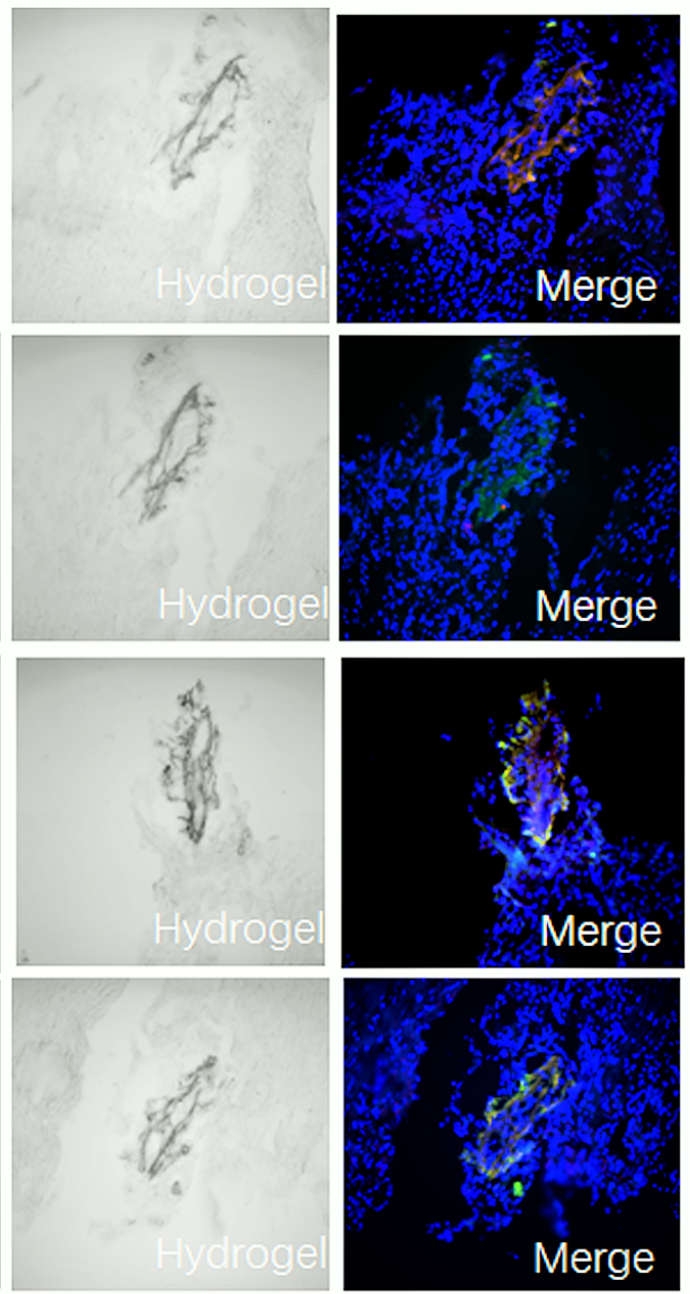

\section{Figure 4}

Differentiation of NSCs in vivo After 7 days of transplantation, the frozen sections were immunohistochemically stained with antibodies against 04, S100, $\beta$ III-tubulin, and GFAP, and imaged by confocal microscopy. Scale bar $=50 \mu \mathrm{m}$. 
A

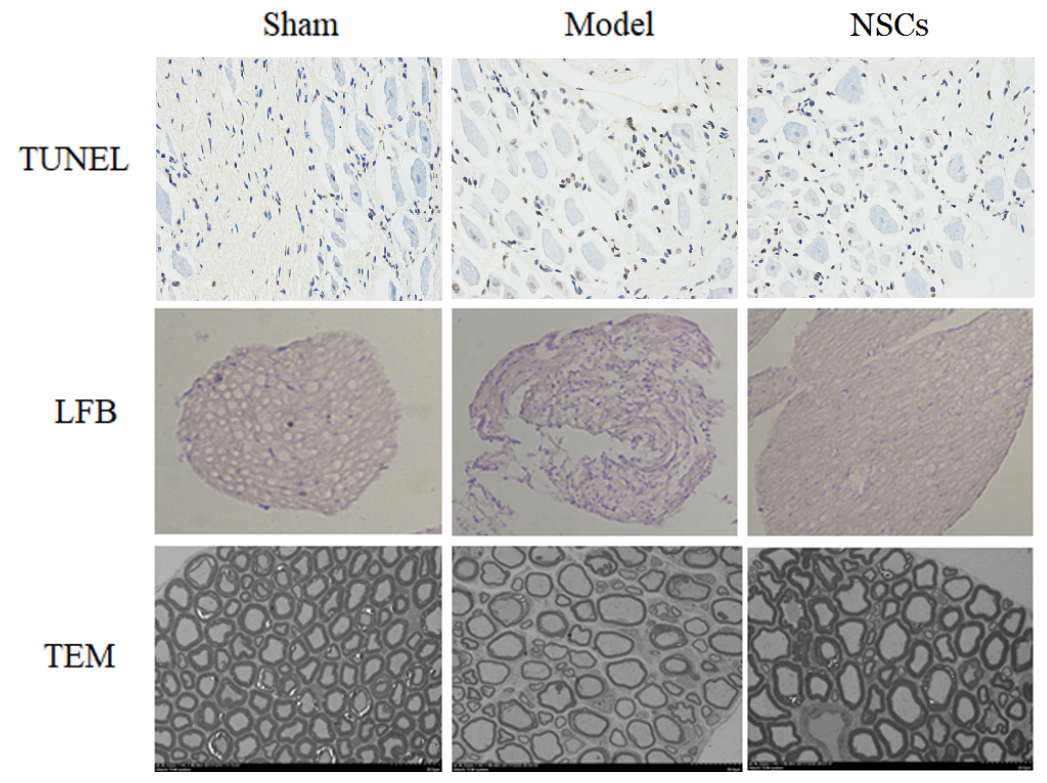

B

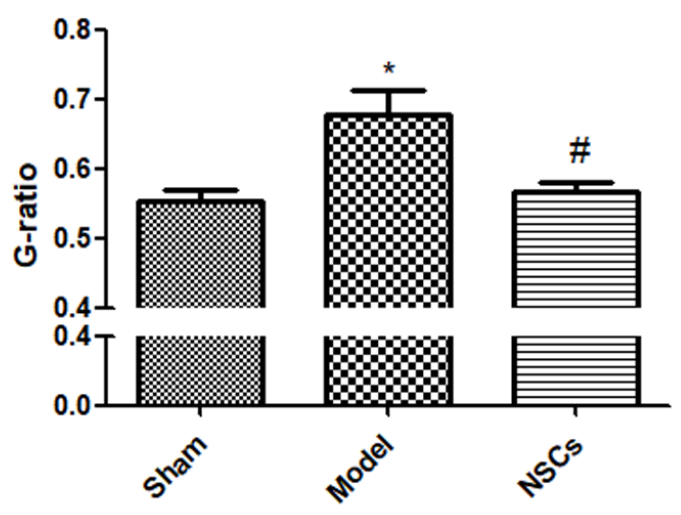

Figure 5

Ultrastructural and pathological changes of DRG after NSC transplantation The rat DRG tissues were subject to TUNEL and LFB staining, and TEM (A). Then, G-ratio (inner diameter/outer diameter of myelinated axons) was calculated (B). ${ }^{*} \mathrm{P}<0.05$, compared to sham group; \# $\mathrm{P}<0.05$, compared to CES model group. Data are reported as means \pm SD.

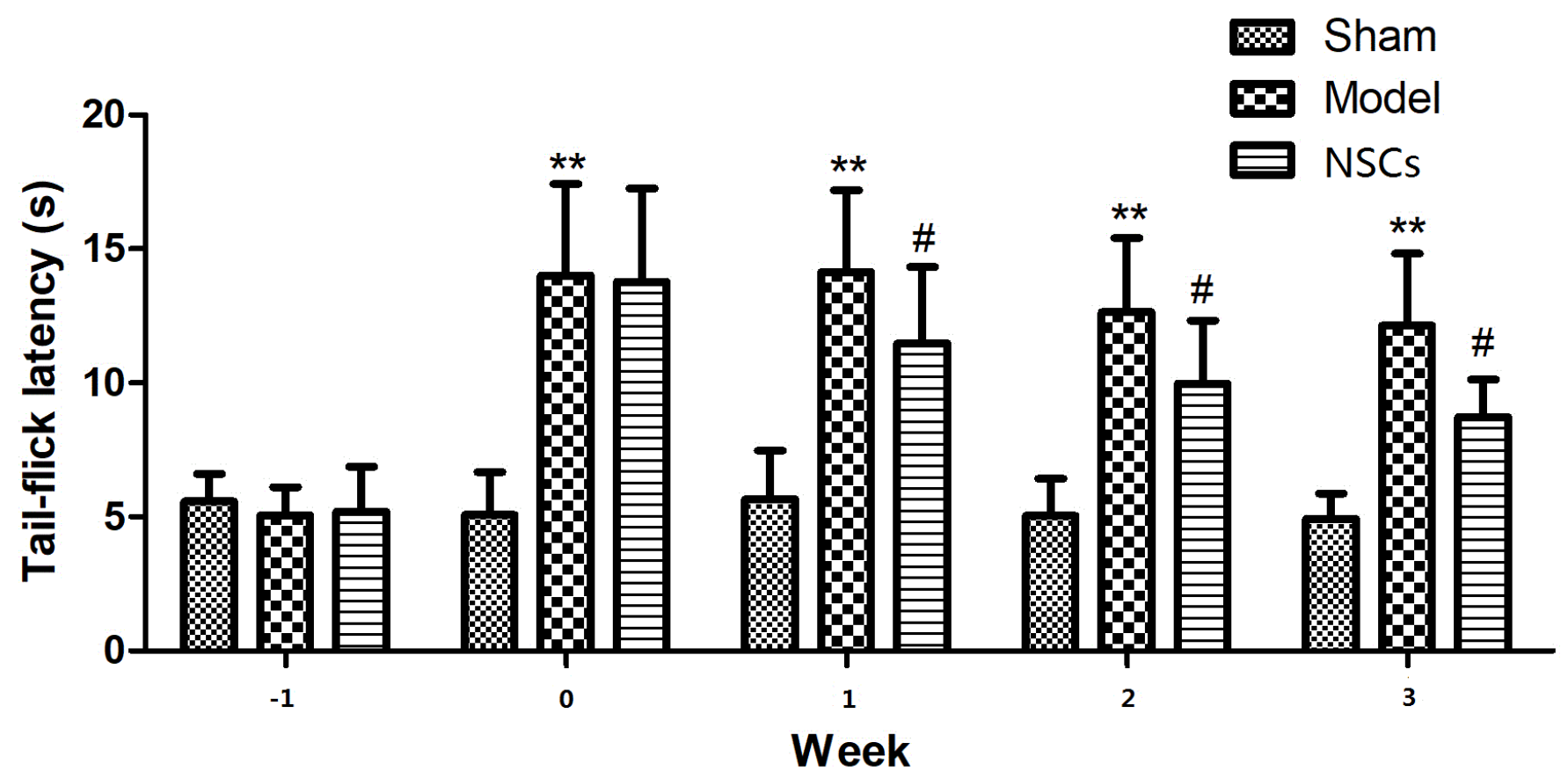

Figure 6 
Functional recovery following transplantation of NSCs encapsulated in hydrogels The functional recovery of sensorimotor reflexes in sham, CES model and NSCs groups was determined using the tail-flick test. ** $\mathrm{P}<0.01$, compared to sham group; \# $\mathrm{P}<0.05$, compared to CES model group. Data are reported as means \pm SD. 